\title{
GypsumFold: Un método para el plegado de paneles de yeso en ángulos variables para la fabricación de productos arquitectónicos
}

\section{GypsumFold: A method for folding gypsum panels at varying angles for the manufacture of architectural products}

\author{
Mauricio Loyola \\ Universidad de Chile, Chile \\ mloyola@uchile.cl \\ Pablo Domínguez \\ Universidad de Chile, Chile \\ ing.pablodominguez@gmail.com \\ Diego Rossel \\ Universidad de Chile, Chile \\ drossel@gmail.com
}

\author{
Nicolás Madariaga \\ Universidad de Chile, Chile \\ n.madariaga.arq@gmail.com \\ Constanza Montiel \\ Universidad de Chile, Chile \\ constanza.montiel@gmail.com
}

\begin{abstract}
Este articulo propone un método para fabricar elementos constructivos con yeso-cartón en ángulos variables una manera fácil, rápida, precisa y económicamente eficiente. Su funcionamiento se basa en el diseño y manufactura automatizada de ranuras fresadas en planchas de yeso-cartón utilizando una técnica innovadora denominada "escalonamiento", la cual permite obtener cualquier ángulo de pliegue usando un mismo procedimiento y herramientas estándar.
\end{abstract}

Keywords: CAD/CAM; Digital Fabrication; Gypsum Board; Complex Geometries

\section{Introducción}

Una tendencia actual en la arquitectura contemporánea es el uso de tecnologías digitales para el diseño y fabricación de productos arquitectónicos con geometrías no ortogonales. Cada vez es más común encontrar formas complejas no sólo en edificios emblemáticos, sino en todo tipo de proyectos, incluyendo tiendas, hoteles, auditorios, edificios corporativos y otros.

Sin embargo, el alto nivel de complejidad de la resolución constructiva de estas formas plantea un importante problema tecnológico. Típicamente, estos productos se fabrican con materiales compuestos (por ejemplo GRG, GRC, GRP) combinados con el uso intensivo de equipos robóticos o $\mathrm{CNC}$, que son de alto costo y complejidad técnica.

En proyectos con recursos limitados, en cambio, la tecnología más extendida para crear geometrías complejas es el uso de planchas o paneles de yeso-cartón (también conocidos como tableros de yeso o placas de yeso), moldeados in situ.

El uso generalizado del yeso-cartón se debe a sus innegables beneficios en términos de costo, comportamiento mecánico y resistencia al fuego. Sin embargo, su proceso constructivo tradicional es lento, intensivo en mano de obra y propenso a errores. Incluso para fabricar ángulos sencillos de $90^{\circ}$ se requiere una cantidad de trabajo considerable. La conformación de aristas para ángulos no tradicionales, como los que se requieren en los diseños contemporáneos, es una tarea tan compleja y sensible en precisión que normalmente se considera del dominio de la artesanía, y por lo tanto, excluida de la industria de la construcción.

La imposibilidad de fabricar adecuadamente productos arquitectónicos geométricamente complejos tiene un impacto negativo en las industrias de diseño, arquitectura y construcción. La consecuencia más evidente es la existencia de productos de baja calidad o ineficientes en material y trabajo, lo que indirectamente impone a los arquitectos una limitación de diseño para espacios no tradicionales.

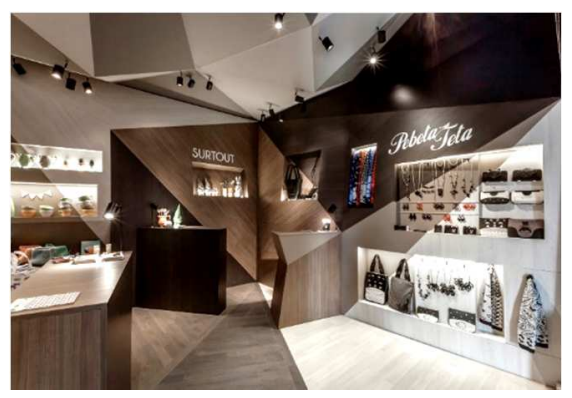

Figura 1: Arriba: Espacio 46, MATEALBINO arquitectura (fuente: www.matealbino.com.ar) 
En respuesta a este problema, este artículo propone una tecnología para la fabricación de productos arquitectónicos mediante el plegado de paneles de paneles de yeso-cartón en ángulos variables con un método fácil, rápido, preciso y económicamente eficiente.

La tecnología se basa en el diseño y manufactura de ranuras fresadas en planchas de yeso-cartón utilizando una técnica propia que hemos denominado "escalonamiento". Por medio de esta técnica, se realizan cortes en el yeso con una fresa plana estándar, milimétricamente controlados en ancho y profundidad, para obtener un ángulo de apertura específico. Al plegar la plancha de yeso-cartón, los vértices de los escalones se tocan, generando un pliegue con el ángulo requerido.

Para el diseño del escalonamiento, el proyecto considera la utilización de un algoritmo propio que determina la geometría de los escalones y define las variables de fresado (cantidad, profundidad, distanciamiento) para cualquier ángulo diédrico que se requiera. Esta solución tecnológica permite al diseñador concentrarse únicamente en el diseño de la forma y geometría del producto constructivo, automatizando la resolución técnica. La manufactura se realiza con un router CNC utilizando fresas planas estándar.

Esta solución tecnológica representa una mejora sustancial frente a las soluciones actuales de plegado de yeso-cartón, las que se basan en la realización de ranuras en forma de $\mathrm{V}$, (normalmente en $90^{\circ}$ ), por lo que el ángulo de pliegue depende estrictamente del ángulo de la fresa, sin posibilidad de variación. No se permiten mayores variaciones que las definidas por la cantidad de fresas disponibles. Por ejemplo, las tecnologías V-Cut ${ }^{\circledR}$ UK, Flextos $\AA \mathrm{GmbH}$, líderes a nivel internacional se basan en este principio y comparten esta limitación. Nuestra tecnología propuesta, en cambio, permite fabricar componentes constructivos con ángulos variables usando un mismo procedimiento y herramienta estándar, sin limitaciones geométricas.

La combinación de las técnicas digitales de diseño y manufactura CNC permite obtener piezas y productos constructivos plegados en yeso-cartón con muchísima mayor libertad formal, con mejor calidad, con menor consumo de material, más rápidas de fabricar e instalar y con resultados significativamente más controlados y precisos de los que se puede intentar obtener hoy en la industria con los métodos tradicionales.

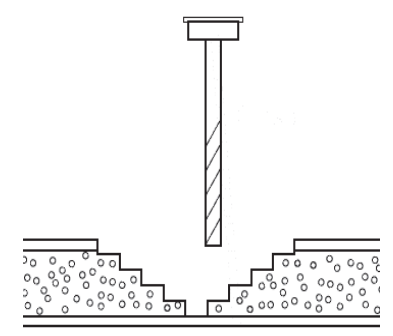

Figura 2a: Principio básico del escalonamiento (fuente: autores).

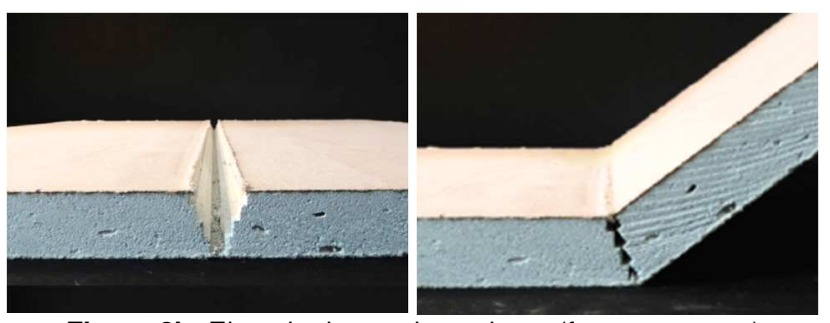

Figura 2b: Ejemplo de escalonamiento (fuente: autores)
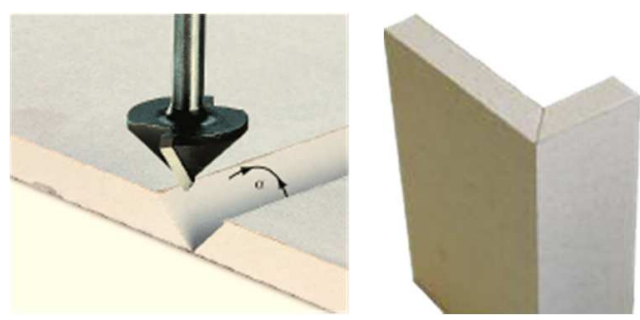

Figura 3: Solución actual en la industria para el plegado de yesocartón. El ángulo de pliegue depende del ángulo de la fresa (usualmente sólo $45^{\circ}, 90^{\circ}$ y $135^{\circ}$ ), sin posibilidad de variación. [fuente imágenes: www.bladesllc.com, www.vcut.co.uk]

\section{Metodología}

El desarrollo de la técnica se realizó en un esquema de 3 objetivos paralelos con actividades independientes y resultados parciales que se retroalimentaban mutuamente.

La primera línea de trabajo fue el desarrollo de la metodología de diseño geométrico de los cortes necesarios y su sistematización en un algoritmo paramétrico digital realizado en Grasshopper 3D.

Luego de un estudio geométrico del problema de escalonamiento, se definieron las variables de entrada y valores de salida requeridos para el algoritmo. La implementación fue trivial, aun cuando se realizaron 14 versiones diferentes del algoritmo en un proceso iterativo de ajuste según se fueron obteniendo los resultados de las otras actividades del proyecto (pruebas de fresado, análisis geométrico angular, pruebas de adhesivos, etc.).

El algoritmo final opera de manera lineal. Como variables de entrada se incluyen la geometría del pliegue deseada (arista base, ángulo de pliegue, terminación de arista), condiciones de material (espesor total del material, espesor del cartón), condiciones de manufactura (diámetro de fresas) y otras variables de ajuste (tolerancias admisibles). Adicionalmente, como variable crítica de diseño se utilizó el número de escalones deseados para cada corte. En este proyecto, este parámetro de diseño fue definido y controlado arbitrariamente por los investigadores como variable independiente, pero se estima que con actividades adicionales de I+D este parámetro debiera derivarse de manera automatizada según de las condiciones de desempeño del producto final. Con las 
variables de entrada y parámetro de diseño, el algoritmo deriva la geometría de los cortes (cantidad de escalones, avance vertical, avance horizontal, terminación de superficie) y entrega como output principal la geometría de fresado en la forma de Rhino Objects (líneas) que son interpretadas como toolpaths en el entorno CAM del router. Posteriormente se decidió incluir un parámetro adicional de profundidad máxima de fresado como criterio de seguridad dependiente de espesor de la fresa.

La segunda línea de trabajo fue la definición de la metodología de fresado CNC. Esta línea resultó notablemente más compleja que la realización de la solución geométrica.

Para el desarrollo de la metodología de fresado se realizaron más de 60 pruebas de fresado $\mathrm{CNC}$ estudiando distintas variables de manufactura. El trabajo se dividió en una primera fase de carácter preliminar y una segunda fase de estudio individual de cada variable (espesores de material, composición de yeso, diámetros y formas de fresas, velocidades de fresado, profundidades de corte, condiciones de remate en pliegues, etc). Cada prueba fue rigurosamente analizada para evaluar su precisión geométrica e integridad estructural mecánica, así como documentación exhaustiva de tiempos de fabricación y observaciones cualitativas.

Entre los varios problemas detectados y subsanados durante este proceso, quizás los principales son los referidos a la terminación de arista superior. Por ejemplo, fue recurrente que el corte del papel cartón superior no siempre se realizó llano y perfecto, sino que con rebabas que afectaban el punto de contacto entre las superficies al conformar el ángulo. Este problema fue solucionado con un pase adicional de la fresa a $1 \mathrm{~mm}$ de profundidad en la zona del papel antes de comenzar el fresado del yeso, que marcaba una línea limpia de corte. Otro ejemplo de problema de manufactura es que cuando se realizaban escalonamientos anchoes, la línea de pliegue tendía a desplazarse y afectar la precisión del ángulo. Este punto fue parcialmente resuelto en el proyecto por medio de un corte en $\mathrm{V}$ que permite tener una arista limpia, pero se trata de una solución que aumenta considerablemente el tiempo de manufactura y por lo tanto resta eficiencia a la tecnología.
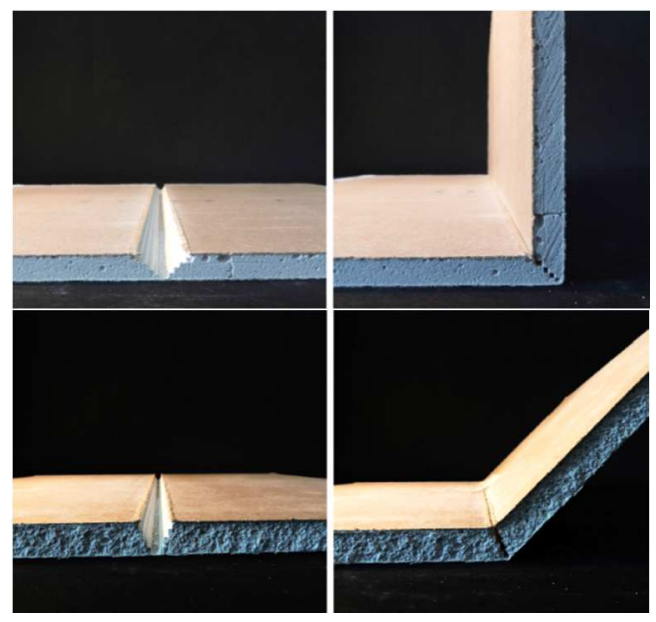

Figura 4: Ejemplos de prototipos preliminares (fuente: autores)
La tercera línea de trabajo fue el estudio de la metodología de conformado, vale decir, de adhesivos para fijar el ángulo.

El principio básico fue la utilización de adhesivos viscosos que también sirvieran como relleno para el espacio vacío que se produce en el producto plegado. De hecho, al tener este espacio, el adhesivo podría fijarse perfectamente sin añadir más holguras ni sacrificar la precisión. Se ensayaron tres adhesivos: basados en yeso, cola de carpintero y epoxi. Se realizaron probetas con diferentes ángulos de pliegue, geometrías de escalonamiento y características de material.

Las probetas con adhesivos fueron luego sometidas a pruebas mecánicas de acuerdo con las normas internacionales y locales. Un hallazgo significativo fue que en todos los casos la fractura ocurrió dentro del yeso y no en el adhesivo o en la superficie de contacto; De modo que la influencia del tipo de adhesivo se evidenció como secundaria en el comportamiento mecánico de las piezas. Los resultados también muestran que el ángulo de plegado es el principal factor incidente sobre la resistencia máxima, incluso sobre la influencia de la geometría de escalonamiento. En un ángulo inferior, mayor es la resistencia. En relación con el comportamiento de la fractura, se pueden distinguir aproximadamente 3 casos. Para las piezas con ángulos agudos $\left(<90^{\circ}\right)$, la ruptura se produce por aplastamiento del borde exterior del panel. En ángulos cercanos a $90^{\circ}$, la ruptura se produce longitudinalmente a la sección del panel. En ángulos obtusos $\left(>90^{\circ}\right)$, la ruptura se produce transversalmente a la sección del panel, perpendicular a sus caras mayores.

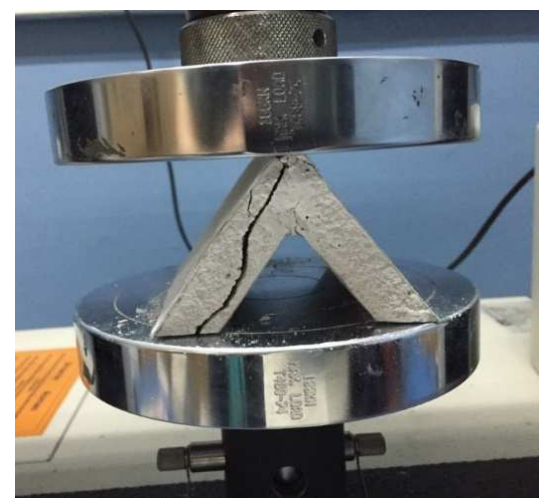

Figura 5: Probeta de ángulo cercano a 90ํe en máquina de ensayo mecánico, con ruptura longitudinal (fuente: autores)

Finalmente, se realizó un ensayo de fabricación de superficies plegadas usando técnicas tradicionales, a modo de control para las posteriores evaluaciones de eficiencia de la técnica propuesta. A un maestro carpintero con experiencia en yesocartón se le solicitó fabricar un pliegue simple de $110^{\circ}$ en una plancha de $50 \times 50 \mathrm{~cm}$ utilizando sus herramientas y técnicas tradicionales. Paralelamente se realizó el mismo pliegue en las mismas dimensiones usando la técnica propuesta en este artículo. Los resultados muestran que para el caso tradicional, el tiempo de fabricación total (sin adhesivo) fue 33 minutos con una desviación de precisión de $15^{\circ}$. Con la técnica de escalonamiento, el tiempo de fabricación (sin adhesivo) fue de $4: 43$ minutos, con una tolerancia inferior a $0.5^{\circ}$. 


\section{Resultados}

Como validación de la hipótesis tecnológica, tres prototipos físicos 1:1 fueron diseñados con pliegues de diferente naturaleza y complejidad. Todos los prototipos incluyeron pliegues en ambos lados del panel ("valles" y "montañas"), representando un reto de precisión de fabricación con la técnica de escalonamiento.

- Prototipo 1, pliegue simple: 40x40cms, yeso-cartón 15mm, 2 pliegues no paralelos, valle y montaña.

- Prototipo 2, pliegue compuesto: $40 \times 40 \mathrm{cms}$, yeso-cartón $15 \mathrm{~mm}, 4$ pliegues convergentes, 2 valles y 2 montanas.

- Prototipo 3, producto arquitectónico: $100 \mathrm{cms}$, yeso-cartón $15 \mathrm{~mm}, 6$ pliegues paralelos de distintas angulaciones, simulando un producto de cielo arquitectónico.

La fabricación se realizó utilizando la técnica exactamente como se presenta en este proyecto. El fresado se realizó con fresas de $3 \mathrm{~mm}$ a $1500 \mathrm{rpm}$, siguiendo las recomendaciones del estudio preliminar. Los cortes fueron precisos y no se hizo daño al yeso o al cartón. La fabricación del vértice de 4 pliegues convergentes (en el prototipo 2) no presentó problemas a pesar de su complejidad, generando un corte limpio en todas las direcciones. Los prototipos fueron fijados con adhesivo epoxi y terminados con 2 capas de pintura blanca. No se necesitó "cinta de unión", relleno, masilla o lijado en ningún momento. Los prototipos fueron instalados como componentes de techo dentro de un espacio arquitectónico, colgado de la estructura de soporte utilizando perfiles de acero galvanizado. Los prototipos no presentaron ninguna deformación ni daños durante su instalación.

El resultado final de todos los prototipos es muy satisfactorio. El nivel de precisión es alto, con una desviación angular respecto al diseño original digital inferior a $0.5^{\circ}$, lo cual cumple con los requerimientos de desempeño fijados como métricas de evaluación de éxito del proyecto. El tiempo de fabricación fue significativamente bajo $(8,11$ y 37 minutos para los prototipos 1, 2 y 3, respectivamente), en comparación con un estándar aproximado de, al menos, 2 a 4 horas, con un método tradicional.

Finalmente, y con el propósito de explorar el eventual campo de aplicación de la tecnología en aplicaciones arquitectónicas, se estudiaron 23 casos de diseño de revestimientos interiores. Los casos fueron agrupados en revestimientos de muros (10 casos), revestimientos de cielos (6 casos), revestimientos de columnas ( 2 casos) y elementos especiales (5 casos). Para cada caso, se analizaron los ángulos comprometidos, el módulo constructivo básico, el patrón de pliegue y su resultado expresivo. Algunos de los casos estudiados se muestran en la figura 8.
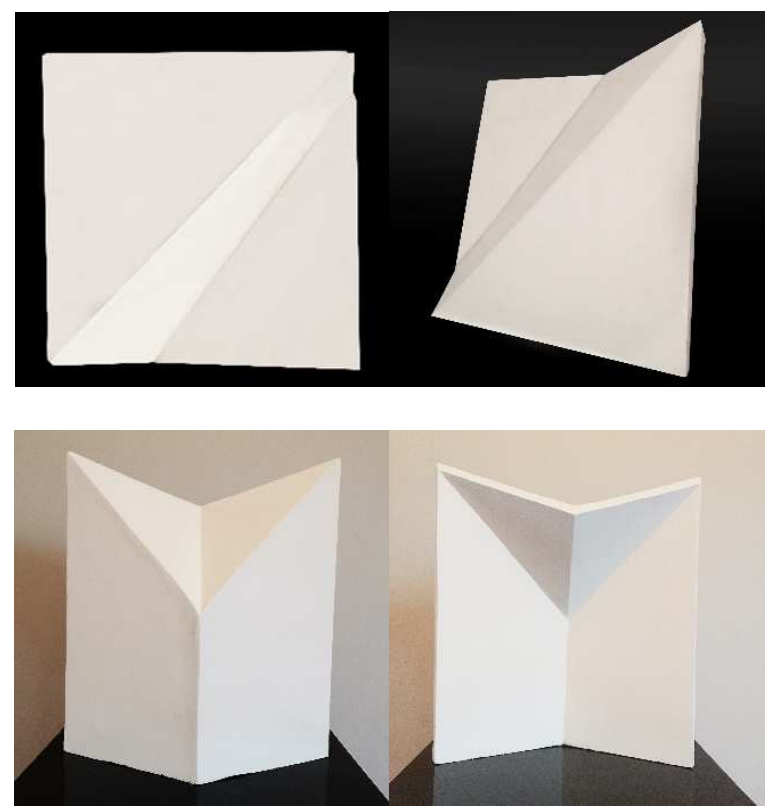

Figura 6: Arriba: Prototipo 1. Abajo: Prototipo 2. (fuente: autores)

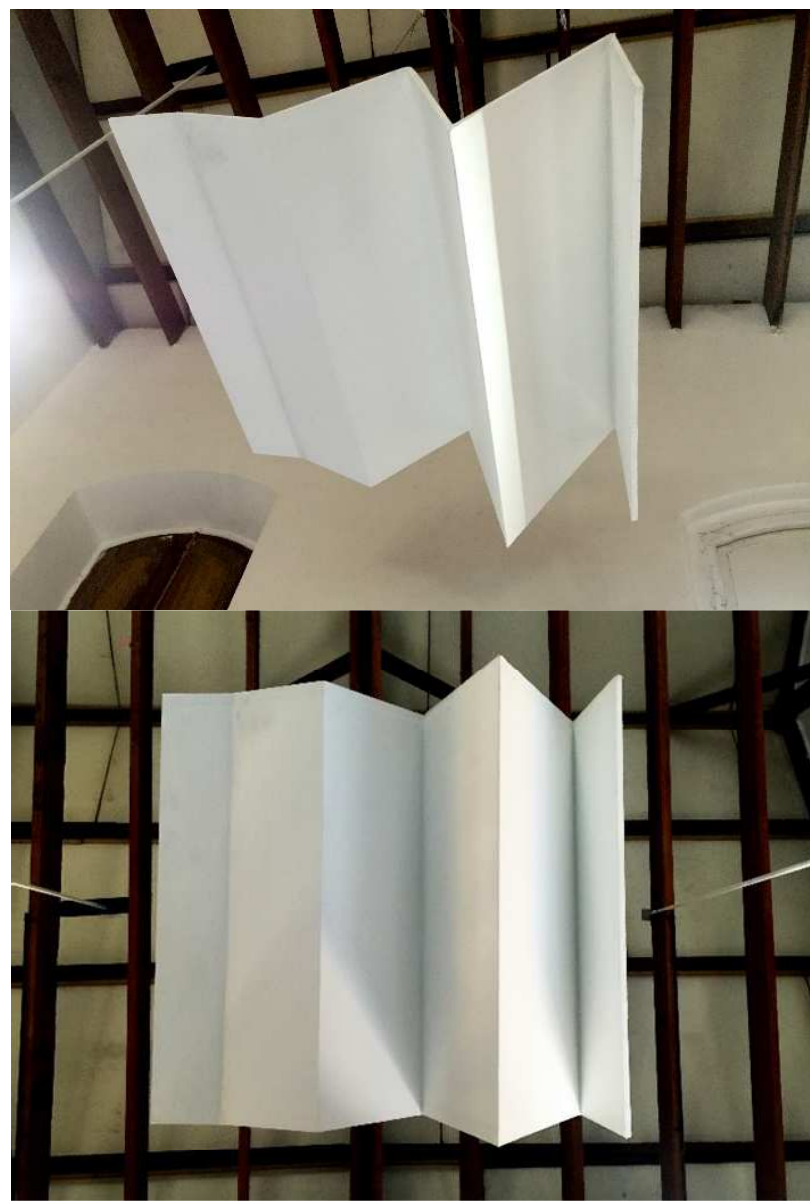

Figura 7: Prototipo 3 (fuente: autores). 

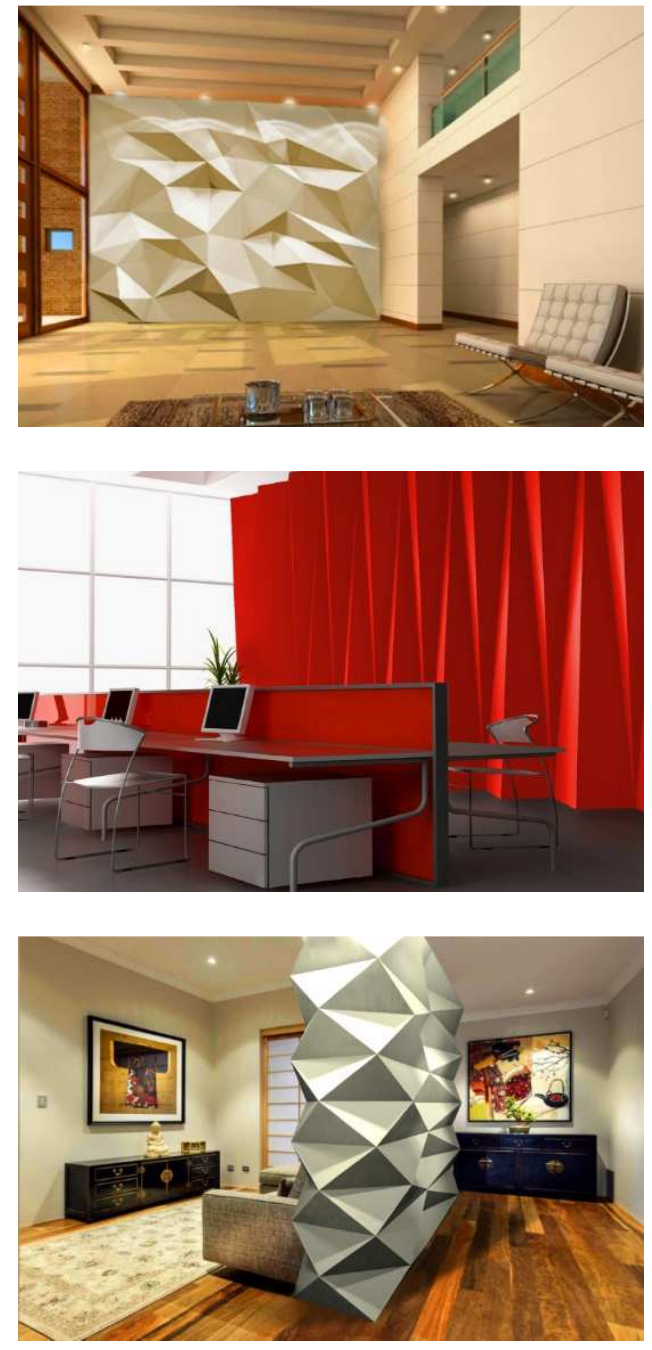

Figura 8: Revestimientos de cielo, revestimientos de columnas y elementos especiales (fuente: autores)

\section{Conclusiones}

Este artículo presenta una innovadora tecnología para la fabricación de productos arquitectónicos de paneles de yeso con ángulos irregulares, validados con prototipos físicos funcionales como prueba de concepto. La tecnología propuesta hace posible fabricar paneles de paneles de yeso plegados con más facilidad, rapidez, precisión y economía que las tecnologías actualmente disponibles en el mercado. Los productos fabricados tienen mayor libertad formal, mejor calidad, menos consumo de material, y se instalan más fácilmente y rápidamente en el sitio. Las pruebas mecánicas preliminares demuestran que los productos cumplen con las regulaciones aplicables.

Sin embargo, el nivel actual de desarrollo de la tecnología no es lo suficientemente maduro para su transferencia al mercado. Es necesario realizar más investigaciones sobre el proceso de fijación y estudiar el uso de otros adhesivos, con el fin de lograr mejores niveles de precisión. También es necesario explorar las posibilidades geométricas de un producto arquitectónico "plegado", por ejemplo, aplicando técnicas de origami. Aun así, es una tecnología prometedora que tiene un potencial interesante para aplicaciones arquitectónicas innovadoras.

\section{Agradecimientos}

Agradecemos a Carlos Cárdenas, María José Lecaros y Víctor Suazo, quienes colaboraron durante el proceso de fabricación y ensayo de los prototipos físicos. Agradecemos también muy especialmente a la Facultad de Arquitectura y Urbanismo que financió esta investigación a través del Fondo FAU 2.0 $01 / 2014$.

\section{Referencias}

Archdaily (2011. 03 de enero). Theatre Agora UNStudio. Extraída el 7 de mayo de 2015 desde http://www.archdaily.com/100224/ theatre-agora-unstudio/photos_iwan-baan_highres_copyright-free_agora-un-lelystad-7071/

Archdaily (2011. 03 de enero). Theatre Agora UNStudio. Extraída el 7 de mayo de 2015 desde http://www.archdaily.com/100224/ theatre-agora-unstudio/photos_iwan-baan_highres_copyright-free_agora-un-lelystad-7071/

Armour Decorative Finishes. (N.D.). Armour Cast, Unique GRG stone mouldings. Obtenido de http://www.adf.com.cy/gpage3. html

Brontis, V. (2010. 8 de noviembre). Construir despliegues: factibilidad material y escalas. Desde http://vladimirbrontis. blogspot.com/2010/11/construir-desplieguesfactibilidad.html

Catálogo Arquitectura. (N.D.). Placas Yeso-Cartón / Knauf. Obtenido de http://www.catalogoarquitectura.cl/placas-yeso-cartonknauf/

EMB Construcción. (2014. Mayo). Knauf Multiform: Formas singulares para trabajos decorativos. Obtenido de http://www. emb.cl/construccion/articulo.mvc?xid=2917\&edi $=141 \&$ xit $=k$ nauf-multiform-formas-singulares-para-trabajosdecorativos

Especificar. (N.D.). Elementos Prefabricados a Medida en Placas de Yeso Cartón D-ZINE. Obtenido de http://www. especificar.cl/fichas/elementos-prefabricados-a-medidaen-placas-de-yeso-carton-d-zine

Festool. (N.D.). Fresa para placas de cartón enyesado HW, vástago 8 $\mathrm{mm}$. Obtenido

https://www.festool.es/Productos/Accesorios/

Pages/Detail.aspx ?pid $=491001 \&$ name $=$ fresa-para-placasde-cart-n-enyesado-hw-s8-d32-90

Grena. A.S. (N.D.). Catálogo FLEXTOS. Obtenido de http://grena. es/wp-

content/uploads/2011/04/Katalog_flextos_Rev4_2_small_ ES.pdf

Institut für Tragkonstruktionen und Konstrucktives Entwerfen. (N.D.). Arboskin: Fassaden Mock-Up aus dauerhaften und rezyklierfähigen Biokunststoffen. Obtenido de http://www.itke.unistuttgart.de/entwicklung.php?id=58\#projekt_bilder

Norma Chilena Oficial. Norma NCh 146/1 Of.2000. Plancha o placas de yeso cartón -parte 1 - Requisitos.

Norma Chilena Oficial. Norma NCh 146/2 Of.2000. Plancha o placas de yeso cartón -parte 2 - Métodos de ensayo. 
SIGraDi 2017, XXI Congreso de la Sociedad Ibero-americana de Gráfica Digital 22 - 24 Noviembre, 2017 - Concepción, Chile.

Romeral. (N.D.). Piezas prefabricadas D-Zine. Obtenido de http://www.romeral.cl/producto.

php?profile $=$ arquitectos $\&$ sistema $=$ dzine $\&$ producto $=10$
USG Boral. (N.D.). Flexiboard® Plasterboard. Obtenido de http://usgboral.com/content/usgcom/en_australia/productssolutions/products/interior 